\title{
Risk Stratification in Paediatric Pulmonary Arterial Hypertension
}

\author{
Shahin Moledina
}

\begin{abstract}
Risk stratification can be defined as clinical assessment and investigation with the purpose of determining a person's risk of suffering a particular outcome and thereby guiding the need for preventative intervention (McGraw-Hill Concise Dictionary of Modern Medicine 2002). The need for risk stratification comes from the realisation that although the presence of pulmonary arterial hypertension significantly impairs survival, the degree of impairment can vary across the population of patients by as much as an order of magnitude.

Furthermore the understanding that therapies aimed at palliating symptoms and prolonging life has negative consequences both for the patients by way of side effects and society by way of costs and should therefore be targeted at those for whom potential benefit outweighs harm.
\end{abstract}

Keywords

Prognosis $\cdot$ Paediatric $\cdot$ Pulmonary hypertension

\subsection{Why Risk Stratify?}

Current drug therapies for pulmonary hypertension are licensed based on randomised control trials primarily demonstrating improvement in exercise intolerance. Currently there are five main therapy classes which may be used in combination, resulting in potentially numerous permutations of therapy. However, with polypharmacy comes a potential

\footnotetext{
S. Moledina $(\triangle)$

United Kingdom National Paediatric Pulmonary Hypertension Service, Great Ormond Street

Hospital, London, UK

e-mail: Shahin.moledina@gosh.nhs.uk
} 
for increased disease burden and side effects. Data from our own centre (unpublished) shows that the number of therapies a patient is receiving negatively impacts health-related quality of life scores even when adjusting for other measures of disease severity. Tools to identify those patients at highest risk of adverse outcome in whom to offer more aggressive therapy are therefore increasingly important. The same is true for interventional procedures used to treat pulmonary hypertension such as balloon atrial septostomy or reverse Potts procedure. Each has a significant procedural mortality between 5 and $15 \%$ which must be balanced against the potential for significant palliation. They therefore should be targeted at those patients who are most likely to benefit.

Finally, cadaveric lung transplantation is a special case where risk stratification is exceedingly important. Lung transplantation especially for younger patients in whom the potential donor pool is restricted, by size and therefore availability, requires anticipation of prognosis. At the time of listing, for a young child in the United Kingdom, for example, might expect to wait a median of 12 months before a suitable matched graft is available. Therefore, if one enters the transplant waiting list too late there is a chance that the patient will succumb to their disease prior to transplantation [1]. Conversely, transplantation itself carries both immediate and longer-term morbidity. The procedure risk is between 5 and $10 \%$, and the median survival is approximately 10 years. Therefore, listing a patient too early in the disease course will serve to impair the outcome and will result in harm.

\subsection{Multidimensional Risk Stratification}

Risk stratification is borne from the observation of discernible differences between patients who experience an early adverse outcome and those who enjoy better longer-term results. These differences span a number of domains and are not always congruous. For example, a patient with very severe vascular disease may have mild symptoms or a patient with mildly increased vascular resistance at assessment may have a particularly aggressive form of disease with rapid progression in vascular disease. Therefore, we proposed a multidimensional assessment of patients to guide risk stratification but also to allow a full understanding of the patient for more holistic treatment and to further understanding of the disease.

\subsection{Factors to Consider in Multidimensional Risk Stratification of children with Pulmonary Arterial Hypertension}

The paradigm of pulmonary arterial hypertension is of vascular remodelling resulting in progressive vascular obstruction and therefore the haemodynamic sequelae of the disease. These processes are thought to result from a combination of inherent susceptibility combined with vascular injury. It is therefore natural to consider the cause of pulmonary hypertension in a particular patient to inform the likely progression of the vascular disease. Since the core of the disease is vascular remodelling, 
measures of vascular structure and function are also considered important in gauging disease severity and risk. Data however indicate that whilst vascular disease is the source of the problem in pulmonary hypertension, outcome is largely mediated by its impact on cardiac function. Finally, as with any chronic disease, the body employs different compensatory mechanisms and therefore a downstream indicator of severity and risk would be the impact of the disease on the patient as a whole.

\subsection{Cause of Pulmonary Hypertension}

It is well known that different aetiologies of pulmonary arterial hypertension have different survival rates [2]. Patients with Eisenmenger syndrome have better survival in most registries than those with idiopathic or postoperative disease. It is likely that patients who develop pulmonary vascular disease despite minimal vascular injury may have more aggressive disease progression compared to those where vascular disease develops only after considerable insult to the vasculature. A prime example of this is the BMPR2 mutation. It has now been demonstrated that patients harbouring a BMPR2 mutation have poorer survival than those with idiopathic pulmonary arterial hypertension without this mutation [3].

\subsection{Vascular Burden}

Since vascular remodelling is central to the disease, measures of remodelling may aid risk stratification. Vascular disease at the microscopic level is difficult to study in patients due to access to tissue, and histology is not routinely performed. Functional cellular assays acting as biomarkers of vascular disease burden have not been well validated and are not in routine clinical use; however, they do represent exciting potential for further research. The classical way of assessing burden of disease in the vasculature in totality is to measure its impact on haemodynamics and, in particular, on pulmonary vascular resistance. As a vessel lumen obstructs the resistance will increase resulting in an aggregate assessment of vascular disease. The ability of pulmonary vascular resistance to predict outcome understandably has been assessed in numerous studies with varying results. A meta-analysis of these data demonstrates a significant association between pulmonary vascular resistance index (PVRI) and outcome with a 32\% increased risk of adverse event for every 10 WU. $\mathrm{m}^{2}$ increase in PVRI [4]. Since the pulmonary vasculature experiences pulsatile blood flow the degree of vascular remodelling cannot be described solely in terms of resistance. The other components of vascular load are total arterial compliance and wave reflection. Wave reflection has not been studied; however, arterial compliance has been investigated to some extent and does predict mortality with the total arterial compliance falling with increased vascular disease. In a number of small studies, pulmonary arterial compliance is seen to be as good as, or better, at predicting outcome than pulmonary vascular resistance. The reason for this is unclear. Finally assessing the acute response of the vascular bed to vasodilators is 
important. In this procedure, patients undergo acute testing of the pulmonary vasodilator, typically inhaled nitric oxide to assess the degree to which the vascular bed will respond by dilating. It is estimated that patients who respond to acute vasodilator testing have about a quarter to a third the risk of patients who do not respond to acute vasodilator testing [4].

As well as assessment of haemodynamics, one can image the vasculature itself with three-dimensional imaging techniques such as computed tomography. In one study CT angiograms from patients with varying degrees of pulmonary hypertension were segmented to look at the complexity of the overall branching structure as a measure of vascular remodelling. In the pilot study, vascular branching complexity correlated both with functional class and pulmonary vascular resistance index but most importantly predicted survival [5].

\subsection{Ventricular Function}

Many of the effects of vascular disease are mediated via its impact on right ventricular function resulting in the observation that patients with very advanced disease, but preserved ventricular function often has a lower symptom burden and better outcome than those with more modest vascular disease but more severe ventricular dysfunction. Ventricular function can be assessed in aggregate during haemodynamic testing by considering total cardiac index which informs prognosis (hazard ratio of 0.66 per $1 \mathrm{~L} / \mathrm{min} / \mathrm{m}^{2}$ increase). Pump failure may also be assessed by measuring right atrial pressure RAP, and this is a robust prognostic indicator with hazard ratio of 1.12 per $1 \mathrm{mmHg}$ increase in RAP. NT-proBNP has been used in various forms of heart failure as a circulating biomarker of ventricular wall stress and dysfunction. Tested in a number of small paediatric studies, this measure also informs prognosis and can be used for risk stratification. One advantage to this measure is that it can be performed in a serial fashion.

Finally the gold standard for measuring cardiac function is cardiac MRI. This can now be performed even for the youngest of children. One study of 100 children undergoing cardiac MRI found a number of measures which are prognostic. These include measures of right ventricular dilatation, severity of tricuspid regurgitation and right ventricular mass; however, the most robust measure in predicting outcome was right ventricular ejection fraction [6].

\subsection{Impact on the Patient}

The most common symptom experienced by patients with pulmonary hypertension is exertional dyspnoea and exercise limitation with nearly $80 \%$ of patients experiencing this [7]. Exercise limitation may be measured by 6-min walking distance; however, numerous studies have failed to demonstrate this as a useful prognostic indicator. A more objective measure of exercise tolerance can be obtained by cardiopulmonary exercise testing, and in an unpublished study from our centre both peak oxygen consumption peak $\mathrm{O}_{2}$ and ventilatory efficiency slope $\left(\mathrm{VE} / \mathrm{VCO}_{2}\right)$ 
were highly prognostic. Perhaps the best way of determining the impact on a patient's ability to function as a result of that disease is to ask them in a structured fashion. This can be scored as in the WHO functional class assessing severity of symptoms. Despite its crude appearance as a subjective score, functional class has been demonstrated consistently to inform prognosis and is a very robust measure. Other ways of assessing impact on patient is to use a structured quality of life questionnaire to score measures of quality of life in different domains. A study from our centre using the PedQL questionnaire has demonstrated good correlation between physical domain of the questionnaire and prognosis.

Finally, children are in the unique position of growing and developing. Chronic diseases impact on this growth and development and therefore measures of growth may be considered as an aggregate measure of the impact on the disease on the patient's biology as a whole. Children with pulmonary hypertension have been shown to have growth failure, and their severity of the growth failure also predicts outcome [7].

\subsection{Summary}

Whilst there is no cure for paediatric pulmonary arterial hypertension at present, treatments have advanced and with more treatment options comes complexity in decision making leading to a need to stratify risk. Numerous markers of adverse outcome have been described across the breadths of domains; however, we believe a multidimensional assessment considering causation, vascular disease burden, cardiac function and overall impact on patient will lead to more accurate risk stratification, promote holistic care of patients and provide deeper insight into disease mechanisms which can later be exploited to therapeutic advantage. Such assessments should be a dynamic process.

\section{References}

1. Lammers AE, Burch M, Benden C, et al. Lung transplantation in children with idiopathic pulmonary arterial hypertension. Pediatr Pulmonol. 2010;45(3):263-9.

2. Haworth SG, Hislop AA. Treatment and survival in children with pulmonary arterial hypertension: the UK Pulmonary Hypertension Service for Children 2001-2006. Heart. 2009;95(4):312-7.

3. Evans JD, Girerd B, Montani D, et al. BMPR2 mutations and survival in pulmonary arterial hypertension: an individual participant data meta-analysis. Lancet Respir Med. 2016;4(2):129-37.

4. Ploegstra MJ, Zijlstra WM, Douwes JM, et al. Prognostic factors in pediatric pulmonary arterial hypertension: A systematic review and meta-analysis. Int J Cardiol. 2015;184:198-207.

5. Moledina S, de Bruyn A, Schievano S, et al. Fractal branching quantifies vascular changes and predicts survival in pulmonary hypertension: a proof of principle study. Heart. 2011;97(15):1245-9.

6. Moledina S, Pandya B, Bartsota M, et al. Prognostic significance of cardiac magnetic resonance imaging in children with pulmonary hypertension. Circ Cardiovasc Imaging. 2013;6(3):407-14.

7. Moledina S, Hislop AA, Foster H, et al. Childhood idiopathic pulmonary arterial hypertension: a national cohort study. Heart. 2010;96(17):1401-6. 
Open Access This chapter is licensed under the terms of the Creative Commons Attribution 4.0 International License (http://creativecommons.org/licenses/by/4.0/), which permits use, sharing, adaptation, distribution and reproduction in any medium or format, as long as you give appropriate credit to the original author(s) and the source, provide a link to the Creative Commons license and indicate if changes were made.

The images or other third party material in this chapter are included in the chapter's Creative Commons license, unless indicated otherwise in a credit line to the material. If material is not included in the chapter's Creative Commons license and your intended use is not permitted by statutory regulation or exceeds the permitted use, you will need to obtain permission directly from the copyright holder.

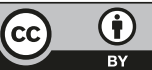

